\title{
Nekrasov's Partition Function and Refined Donaldson-Thomas Theory: the Rank One Case
}

\author{
Balázs SZENDRŐI
}

Mathematical Institute, University of Oxford, UK

E-mail: szendroi@maths.ox.ac.uk

URL: http://people.maths.ox.ac.uk/szendroi/

Received June 12, 2012, in final form November 05, 2012; Published online November 17, 2012

http://dx.doi.org/10.3842/SIGMA.2012.088

\begin{abstract}
This paper studies geometric engineering, in the simplest possible case of rank one (Abelian) gauge theory on the affine plane and the resolved conifold. We recall the identification between Nekrasov's partition function and a version of refined DonaldsonThomas theory, and study the relationship between the underlying vector spaces. Using a purity result, we identify the vector space underlying refined Donaldson-Thomas theory on the conifold geometry as the exterior space of the space of polynomial functions on the affine plane, with the (Lefschetz) SL(2)-action on the threefold side being dual to the geometric SL(2)-action on the affine plane. We suggest that the exterior space should be a module for the (explicitly not yet known) cohomological Hall algebra (algebra of BPS states) of the conifold.
\end{abstract}

Key words: geometric engineering; Donaldson-Thomas theory; resolved conifold

2010 Mathematics Subject Classification: 14J32

\section{Introduction}

We study an instance of geometric engineering [14]. Our starting point is Type II string theory on a real 10-dimensional spacetime $X \times \mathbb{C}^{2}$, the product of the flat Calabi-Yau surface $\mathbb{C}^{2}$ and a local Calabi-Yau threefold $X$. This threefold is determined by a finite subgroup $\Gamma<\mathrm{SU}(2)$; it is the minimal resolution $X \rightarrow \mathcal{O}_{\mathbb{P}^{1}}(-1,-1) / \Gamma$ of the singular threefold $\mathcal{O}_{\mathbb{P}^{1}}(-1,-1) / \Gamma$, where $\Gamma$ acts fiberwise on the resolved conifold $\mathcal{O}_{\mathbb{P}^{1}}(-1,-1)$, fixing the zero-section. Integrating out the $X$-direction leads to supersymmetric gauge theory on $\mathbb{C}^{2}$ with gauge group $G=G(\Gamma)$, the simple group of type A, D or E corresponding to the type of $\Gamma$. On the other hand, integrating out the $\mathbb{C}^{2}$-direction gives a $\sigma$-model, or equivalently a version of $U(1)$ gauge theory on $X$.

The aim of this paper is to revisit the construction of the partition functions $Z_{\mathbb{C}^{2}}$ and $Z_{X}$ on the two sides and their identification, mainly in the simplest possible case when $\Gamma$ is the trivial group. As it turns out, both partition functions are characters of representations of tori (Hilbert series of graded vector spaces). The underlying vector spaces are the symmetric, respectively the exterior space of the space of functions on $\mathbb{C}^{2}$. On the four-dimensional side this is not new, but it is a surprising fact on the six-dimensional side, and suggests that a version of Koszul duality might lurk behind the mathematics of geometric engineering. It would be very interesting to see more examples in action to substantiate this claim; we comment on the difficulties below. Slightly more concretely, we suggest at the end of the paper that the alternating space $\Lambda^{*} \mathbb{C}[x, y]$ should be a module for the Kontsevich-Soibelman CoHA (cohomological Hall algebra) $\mathcal{H}(Q, W)$ attached to the conifold quiver $(Q, W)$; an explicit description of this algebra is currently lacking but would be desirable.

\footnotetext{
*This paper is a contribution to the Special Issue "Mirror Symmetry and Related Topics". The full collection is available at http://www.emis.de/journals/SIGMA/mirror_symmetry.html
} 


\section{The four-dimensional partition function}

While it would be possible to remain more general for a while at least, let us make a simplifying assumption right away and assume that $\Gamma$ is the cyclic group of order $r$, embedded diagonally into $\mathrm{SU}(2)$. Thus, on the four-dimensional side we are considering $\mathrm{SU}(r)$ gauge theory on the complex plane $\mathbb{C}^{2}$. This is certainly well-defined for $r>1$; for $r=1$ we recall the interpretation below.

The partition function of the theory is a sum of integrals over a collection of moduli spaces, the spaces of $\mathrm{SU}(r)$ instantons on $\mathbb{R}^{4}$ of various charges $k$. It is well known that, after framing the instantons, their moduli space is diffeomorphic to the moduli spaces

$$
\mathcal{M}_{r, k}^{\circ}=\left\{\begin{array}{l|l}
(\mathcal{E}, \varphi) \mid \begin{array}{l}
\mathcal{E} \text { vector bundle on } \mathbb{P}^{2} \\
\operatorname{rk} \mathcal{E}=r, c_{2}(\mathcal{E})=k, \varphi:\left.\mathcal{E}\right|_{l_{\infty}} \cong \mathcal{O}_{l_{\infty}}^{\oplus r}
\end{array}
\end{array}\right\} / \sim
$$

of framed rank-r bundles $\mathcal{E}$ on $\mathbb{P}^{2}$ of charge $k$. Here $l_{\infty} \cong \mathbb{P}^{1}$ is the complement of $\mathbb{C}^{2}$ in $\mathbb{P}^{2}$. Note that the parametrized bundles $\mathcal{E}$ are indeed $\mathrm{SL}(r)$-bundles, since the framing isomorphism automatically trivializes the determinant. This space admits two partial compactifications $\mathcal{M}_{r, k}$ and $\overline{\mathcal{M}}_{r, k}$. First, we have the space

$$
\mathcal{M}_{r, k}=\left\{\begin{array}{l|l}
(\mathcal{E}, \varphi) & \begin{array}{l}
\mathcal{E} \text { torsion-free sheaf on } \mathbb{P}^{2} \\
\operatorname{rk} \mathcal{E}=r, c_{2}(\mathcal{E})=k, \varphi:\left.\mathcal{E}\right|_{l_{\infty}} \cong \mathcal{O}_{l_{\infty}}^{\oplus r}
\end{array}
\end{array}\right\} / \sim
$$

the moduli space of framed rank-r torsion-free sheaves on $\mathbb{P}^{2}$ of charge $k$; this is the analogue of the Gieseker compactification of the moduli of bundles for a projective surface. $\mathcal{M}_{r, k}$ is a nonsingular holomorphic sympletic variety of dimension $2 k r$. The second space $\overline{\mathcal{M}}_{r, k}$ is the analogue of the Uhlenbeck compactification, and can be constructed as an affine GIT quotient; for details, see [22, Section 2].

The four-dimensional gauge theoretic partition function (for pure gauge theory, in the absence of matter) is

$$
Z_{\mathbb{C}^{2}, r}(\Lambda)=\sum_{k \geq 0} \Lambda^{k} \int_{\mathcal{M}_{r, k}} 1,
$$

the generating function of symplectic volumes of the (non-singular) moduli spaces $\mathcal{M}_{r, k}$. This is an ill-defined expression, since 1 is not a top-dimensional form, and integration happens over noncompact spaces $\mathcal{M}_{r, k}$.

As Nekrasov [24] discovered, one can "renormalize" both these problems by considering equivariant integration with respect to the torus $T=\left(\mathbb{C}^{*}\right)^{2} \times\left(\mathbb{C}^{*}\right)^{r-1}$. Here the first factor acts via its geometric action on $\mathbb{C}^{2}$, which extends to an action on $\mathcal{M}_{r, k}$. The second component $\left(\mathbb{C}^{*}\right)^{r-1}$, the maximal torus of $\mathrm{SL}(r)$, acts on the framing $\varphi$, fixing the trivialization of the determinant.

We will in fact be interested in a K-theoretic version ${ }^{1}$ of the partition function, also introduced in [24] and studied in detail in [22, 23]. In the K-theoretic version, the integrand 1 gets interpreted as the unit K-theory class, the class of the structure sheaf $\mathcal{O}$; integration gets replaced by pushforward to the point. Thus, the partition function computes the generating series of equivariant coherent cohomologies of $\mathcal{O}$. This sort of generating series had been considered earlier in a related context under the name of four-dimensional Verlinde formula in [18].

Introducing a basis $q_{1}, q_{2}, a_{1}, \ldots, a_{r-1}$ for the space of $T$-characters, the $K$-theoretic partition function is

$$
Z_{\mathbb{C}^{2}, r}\left(q_{i}, a_{j}, \Lambda\right)=\sum_{k \geq 0} \Lambda^{k} \operatorname{char}_{T} R\left(\pi_{r, k}\right)_{*} \mathcal{O}_{\mathcal{M}_{r, k}} \in \mathbb{Z}\left(q_{i}, a_{j}\right)[[\Lambda]] .
$$

\footnotetext{
${ }^{1}$ What I call $Z_{\mathbb{C}^{2}, r}$ is in fact called the 5-dimensional partition function in the physics literature, since it arises from studying M-theory on a circle bundle over $X \times \mathbb{C}^{2}$. I will abuse language and will continue to call it the four-dimensional partition function, since it is still naturally associated to the complex surface.
} 
Here $\pi_{r, k}: \mathcal{M}_{r, k} \rightarrow\{*\}$ is the structure morphism, $\operatorname{char}_{T} V$ denotes the $T$-character of a representation $V$ of the torus $T$, and $\operatorname{char}_{T} R\left(\pi_{r, k}\right)_{*}$ is shorthand for $\sum_{i}(-1)^{i} \operatorname{char}_{T} R^{i}\left(\pi_{r, k}\right)_{*}$, the torus character of an (a priori) virtual representation of $T$. The formula (2) gives a well-defined expression, since it is easy to see that each $T$-weight space of each $R^{i}\left(\pi_{r, k}\right)_{*} \mathcal{O}_{\mathcal{M}_{r, k}}$ is finite dimensional [22, Section 4]. It can be shown that the answer is indeed a rational function of the variables $q_{i}, a_{j}$.

It is known that $\pi_{r, k}$ factors through a map $\sigma_{r, k}: \mathcal{M}_{r, k} \rightarrow \overline{\mathcal{M}}_{r, k}$ to the Uhlenbeck-type space. Pushforward under $\sigma_{r, k}$ gives no higher cohomology [22, Lemma 3.1]; also $\overline{\mathcal{M}}_{r, k}$ is affine, so there is no higher cohomology there either. Hence the partition function is simply

$$
Z_{\mathbb{C}^{2}, r}\left(q_{i}, a_{j}, \Lambda\right)=\sum_{k \geq 0} \Lambda^{k} \operatorname{char}_{T} H^{0}\left(\overline{\mathcal{M}}_{r, k}, \mathcal{O}_{\overline{\mathcal{M}}_{r, k}}\right) \in \mathbb{Z}\left(q_{i}, a_{j}\right)[[\Lambda]]
$$

Let us restrict further to the case $r=1$, which in any case requires some further explanation. In this case we have "SU(1) gauge theory" on $Y=\mathbb{C}^{2}$. This gets interpreted as the theory of framed rank-one sheaves with trivial determinant on $\mathbb{P}^{2}$. Since there is only one line bundle on $\mathbb{P}^{2}$ with trivial determinant, the moduli space $\mathcal{M}_{1, k}$ of framed rank-one torsion-free sheaves on $\mathbb{P}^{2}$ can be identified with Hilb ${ }^{k}\left(\mathbb{C}^{2}\right)$, the Hilbert scheme of $k$ points on $\mathbb{C}^{2}$. The corresponding Uhlenbeck space $\overline{\mathcal{M}}_{1, k}$ is the symmetric product $S^{k}\left(\mathbb{C}^{2}\right) ; \sigma_{1, k}$ is the Hilbert-Chow morphism. There are no $a_{j}$ parameters. The partition function in this case can be computed explicitly in closed form.

Proposition 2.1 ([22, 25]). We have

$$
Z_{\mathbb{C}^{2}, r=1}\left(q_{1}, q_{2}, \Lambda\right)=\prod_{i_{1}, i_{2} \geq 0}\left(1-q_{1}^{i_{1}} q_{2}^{i_{2}} \Lambda\right)^{-1}
$$

Proof.

$$
\begin{aligned}
Z_{\mathbb{C}^{2}, r=1}\left(q_{1}, q_{2}, \Lambda\right) & =\sum_{k \geq 0} \Lambda^{k} \operatorname{char}_{T} H^{0}\left(\mathcal{O}_{S^{k}\left(\mathbb{C}^{2}\right)}\right)=\sum_{k \geq 0} \Lambda^{k} \operatorname{char}_{T} \mathbb{C}\left[x_{1}, \ldots, x_{k}, y_{1}, \ldots, y_{k}\right]^{S_{k}} \\
& =\sum_{k \geq 0} \Lambda^{k} \operatorname{char}_{T} S^{k} \mathbb{C}[x, y]=\operatorname{char}_{T \times \mathbb{C}^{*}} S^{*} \mathbb{C}[x, y]=\prod_{i_{1}, i_{2} \geq 0}\left(1-q_{1}^{i_{1}} q_{2}^{i_{2}} \Lambda\right)^{-1} .
\end{aligned}
$$

Here in the penultimate line, $S^{*} \mathbb{C}[x, y]$ is treated as a triply-graded space, graded by $x$-weight, $y$-weight and polynomial weight with respect to the outer symmetric power operation. A triple grading corresponds to an action of a rank-three torus $T \times \mathbb{C}^{*}$, and the character is the Hilbert series.

In the higher rank case, there is no known closed formula for $Z_{\mathbb{C}^{2}, r}\left(q_{i}, a_{j}, \Lambda\right)$. Through torus localization, it can be computed as a sum over the $T$-fixed points of the spaces $\mathcal{M}_{r, k}$, parametrized by $r$-tuples of partitions [24, 25]. It is shown in [23] that it satisfies a system of functional equations called the blowup equations, whose solution is unique.

\section{The six-dimensional partition function}

Under geometric engineering, the four-dimensional partition function $Z_{\mathbb{C}^{2}, r}$ should correspond to a partition function built out of invariants of the Calabi-Yau threefold $X$. The gauge group on the threefold $X$ is always going to be $\mathrm{SU}(1)$, in other words we will be looking at a version of rank one sheaf theory with trivial determinant. To find out precisely which version, let us restrict to the case $r=1$ again. 
From the four-dimensional theory we obtain the partition function $Z_{\mathbb{C}^{2}, r=1}\left(\Lambda, q_{1}, q_{2}\right)$. Taking its inverse and specializing, consider

$$
Z(q, T)=Z_{\mathbb{C}^{2}, r=1}\left(\Lambda=-T q, q_{1}=q_{2}=-q\right)^{-1}=\prod_{n \geq 1}\left(1-(-q)^{n} T\right)^{n} .
$$

This is a very well-known expression, the reduced topological string partition function [9] of the resolved conifold $X=\mathcal{O}_{\mathbb{P}^{1}}(-1,-1)$. After a further change of variables [19], the function $\log Z\left(q=-e^{i \hbar}, T\right)$ gives the full Gromow-Witten potential of the resolved conifold $X$, with $\hbar$ being the genus parameter.

On the other hand, the precise geometric interpretation of the coefficients $Z(q, T)$ is given by

Theorem 3.1 ([21]). We have

$$
\prod_{n \geq 1}\left(1-(-q)^{n} T\right)^{n}=\sum_{l, m \geq 0} P_{l, m} T^{l} q^{m}
$$

where $P_{l, m}$ are the pairs invariants or Pandharipande-Thomas (PT) invariants [28] of the resolved conifold $X$.

Let us recall from [28] how the invariants $P_{l, m}$ are defined. They are the enumerative invariants associated to a collection of highly singular moduli spaces $\mathcal{N}_{l, m}$, which carry a perfect obstruction theory. These spaces are the moduli spaces of stable pairs

$$
\mathcal{N}_{l, m}=\left\{\begin{array}{l|l}
(\mathcal{F}, s) & \begin{array}{l}
\mathcal{F} \text { pure 1-dimensional sheaf with proper support on } X \\
s: \mathcal{O}_{X} \rightarrow \mathcal{F} \text { a section } \\
\operatorname{Supp}(\mathcal{F})=l\left[\mathbb{P}^{1}\right], \chi(\mathcal{F})=m, \operatorname{dim} \operatorname{Supp} \text { coker }(s)=0
\end{array}
\end{array}\right\} / \sim,
$$

where the restriction of the cokernel of $s$ having zero-dimensional support is the stability condition. Note that the sheaf $\mathcal{F}$, having necessarily proper support, must be supported on a multiple of the zero-section in $X$. As observed by Bridgeland [3], the spaces $\mathcal{N}_{l, m}$ represent a moduli problem involving perverse coherent sheaves on $X$. Perverse coherent sheaves are complexes of coherent sheaves, which belong to the heart of a $t$-structure on the derived category of sheaves on $X$ different from the standard one.

Our aim is to find a one-parameter refinement of the numbers $P_{l, m}$, in order to obtain a refinement of the topological string partition function of the conifold which matches the full Nekrasov's formula. The following result is crucial for further progress.

Theorem 3.2. The moduli spaces $\mathcal{N}_{l, m}$ are proper. Moreover, they are global degeneracy loci: there exist smooth varieties $N_{l, m}$ equipped with regular functions $f_{l, m}: N_{l, m} \rightarrow \mathbb{C}$ so that, schemetheoretically,

$$
\mathcal{N}_{l, m}=\left\{d f_{l, m}=0\right\} \subset N_{l, m}
$$

are the degeneracy loci of these functions.

Proof. The first statement follows from the fact that the only proper curve in $X=\mathcal{O}_{\mathbb{P}^{1}}(-1,-1)$ is the zero section, so the reduced support of a stable pair cannot move.

To prove the second statement, we need to recall the quiver interpretation of the moduli spaces attached to the resolved conifold [30]. First of all, let $Q$ be the conifold quiver, consisting of vertex set $V=\{0,1\}$ and edge set $\left\{a_{01}, b_{01}, a_{10}, b_{10}\right\}$, with edges labelled $i j$ pointing from vertex $i$ to vertex $j$. Consider the superpotential [15]

$$
W=a_{01} a_{10} b_{01} b_{10}-a_{01} b_{10} b_{01} a_{10} .
$$


Let also $\widetilde{Q}$ be the framed (or extended) quiver with vertex set $\widetilde{V}=\{0,1, \infty\}$, an extra edge $i_{\infty 0}$ and the same superpotential $\widetilde{W}=W$. Then by [21], the moduli spaces $\mathcal{N}_{l, m}$ can be identified with stable representations of the quiver $\widetilde{Q}$ with relations arising from formal partial derivatives of $\widetilde{W}$ with respect to the various edges, and a dimension vector $\mathbf{d}=\left(d_{0}, d_{1}, d_{\infty}=1\right)$ where $\left(d_{0}, d_{1}\right)$ depends on $l, m$. Stability is taken with respect to a specific stability condition, determined by a particular (limiting) value of a stability parameter $\theta \in \mathbb{R}^{V}$ (see more on this below).

Now let $N_{l, m}$ be the moduli space of $\theta$-stable representations of the quiver $\widetilde{Q}$ with the same dimension vector $\mathbf{d}$, but with no relations. Since $\theta$ is chosen generically, this is a smooth quasiprojective variety. Let also $f_{l, m}: N_{l, m} \rightarrow \mathbb{C}$ be defined by evaluating the expression $\operatorname{Tr}(W)$ on representations. It is now well known that the scheme-theoretic equations of $\mathcal{N}_{l, m} \subset N_{l, m}$ are indeed given by $d \operatorname{Tr}(W)=0$.

Remark 3.3. There is a further point worth mentioning in connection with this construction. As well as the smooth GIT quotient $N_{l, m}$, there is also the affine GIT quotient $\bar{N}_{l, m}$ together with a contraction morphism $N_{l, m} \rightarrow \bar{N}_{l, m}$ (an analogue of the map $\sigma_{r, k}$ in the four-dimensional situation). This is a projective morphism, equipped by construction with a relatively ample line bundle (coming from the choice of GIT stability). On the other hand, $\mathcal{N}_{l, m}$, being proper, must sit in a fibre of this contraction. Thus $\mathcal{N}_{l, m}$ comes automatically equipped with a polarization, a chosen ample line bundle $\mathcal{L}_{l, m}$.

Theorem 3.2 implies that the singular moduli space $\mathcal{N}_{l, m}$ acquires a topological coefficient system

$$
\varphi_{l, m}=\varphi_{f_{l, m}} \mathbb{Q}_{N_{l, m}}\left[\operatorname{dim} N_{l, m}\right] \in \operatorname{Perv}_{\mathbb{Q}}\left(\mathcal{N}_{l, m}\right),
$$

the perverse sheaf of vanishing cycles of the regular function $f_{n, l}$ on $N_{l, m}$. This perverse $\mathbb{Q}$-sheaf is well known to live on the (reduced) degeneracy locus. In fact, we wish to use Hodge theory, so we consider the canonical lift

$$
\Phi_{l, m}=\varphi_{f_{n, l}}^{H} \mathbb{Q}_{N_{l, m}}^{H}\left(\operatorname{dim} N_{l, m} / 2\right)\left[\operatorname{dim} N_{l, m}\right] \in \widetilde{\operatorname{MHM}}_{\mathbb{Q}}\left(\mathcal{N}_{l, m}\right),
$$

the mixed Hodge module of vanishing cycles of $\varphi_{f, n}$. To make sense of the above expression, we need to extend the category of mixed Hodge modules by a half-Tate object; see Appendix A.

Now consider the (hyper)cohomology $\mathbb{H}^{*}\left(\mathcal{N}_{l, m}, \Phi_{l, m}\right)$. As the cohomology of a mixed Hodge module, it carries a weight filtration $W_{n}$. Consider the weight polynomial ${ }^{2}$ (for details and examples, see Appendix A again)

$$
W\left(\mathcal{N}_{l, m}, \Phi_{l, m} ; t^{\frac{1}{2}}\right)=\sum_{n \in \mathbb{Z}} t^{\frac{n}{2}} \sum_{i \in \mathbb{Z}}(-1)^{i} \operatorname{dim} \mathrm{Gr}_{n}^{W} \mathbb{H}^{i}\left(\mathcal{N}_{l, m}, \Phi_{l, m}\right) \in \mathbb{Z}\left[t^{ \pm \frac{1}{2}}\right]
$$

Here $\operatorname{Gr}_{n}^{W}$ denotes the $n$-th graded piece under the weight filtration. Note that the weight polynomial is usually defined on compactly supported cohomology, but this makes no difference here, as $\mathcal{N}_{l, m}$ is proper, and $\Phi_{l, m}$ is self-dual under Verdier duality.

The following result shows that we are indeed considering here a one-parameter refinement of the numerical invariants introduced earlier.

Proposition 3.4. We have

$$
P_{l, m}=W\left(\mathcal{N}_{l, m}, \Phi_{l, m} ; t^{\frac{1}{2}}=1\right) .
$$

\footnotetext{
${ }^{2}$ We assume here for simplicity of exposition that the semisimple part of the monodromy acts trivially on the cohomology, as will be the case in the example where we apply this formalism. In general, the weight filtration needs to be shifted on the part of the cohomology where the semisimple monodromy acts nontrivially.
} 
Proof. Setting $t^{\frac{1}{2}}=1$ in the weight polynomial, we are ignoring the weight filtration, and thus computing the Euler characteristic of the perverse sheaf of vanishing cycles of the function $f_{l, m}$ on $N_{l, m}$. As discussed in [1], this computes the integral (weighted Euler characteristic) of a canonical constructible function, the so-called Behrend function, on the degeneracy locus $\mathcal{N}_{l, m}$. On the other hand, the numerical invariant $P_{l, m}$ arises from a symmetric perfect obstruction theory on $\mathcal{N}_{l, m}$. By the main result of [1], the degree of the associated virtual fundamental class agrees with the Euler characteristic of $\mathcal{N}_{l, m}$ weighted by the Behrend function.

Consider the generating series

$$
Z_{X}\left(q, T, t^{\frac{1}{2}}\right)=\sum_{l, m \in \mathbb{Z}} W\left(\mathcal{N}_{l, m}, \Phi_{l, m} ; t^{\frac{1}{2}}\right) T^{l} q^{m} .
$$

This series is computed in the following result.

Theorem 3.5 ([20]). We have

$$
Z_{X}\left(q, T, t^{\frac{1}{2}}\right)=\prod_{m \geq 1} \prod_{j=0}^{m-1}\left(1-(-1)^{m} t^{-\frac{m}{2}+\frac{1}{2}+j} q^{m} T\right) .
$$

Remark 3.6. Our paper [20] works in a slightly different framework, considering a ring-valued rather than cohomological invariant, taking values in a version of the motivic ring of varieties, thus adopting in the approach of [17]. However, there is a homomorphism from the motivic ring to the polynomial ring $\mathbb{Z}\left[t^{ \pm \frac{1}{2}}\right]$, given by the weight polynomial, since the weight polynomial respects the fundamental defining relation $[X]=[X \backslash Z]+[Z]$ of the motivic ring, where $Z \subset X$ is a closed subvariety. Hence the results of [20] indeed apply.

Thus, comparing (4) with (6), we obtain a full interpretation of (the inverse of) the Nekrasov partition function in the simplest case $r=1$ :

Corollary 3.7. The four-dimensional and six-dimensional generating series are related by the equality

$$
Z_{\mathbb{C}^{2}, r=1}\left(q_{1}, q_{2}, \Lambda\right)=Z_{X}\left(q, T, t^{\frac{1}{2}}\right)^{-1}
$$

under the change of variables $q_{1}=-t^{\frac{1}{2}} q, q_{2}=-t^{-\frac{1}{2}} q, \Lambda=-T q$.

Remark 3.8. The search for, and development of, a refined version of Donaldson-Thomas theory has always been motivated and informed by the relationship to Nekrasov's partition function $[11,12,13]$. The fact that the motivic, or equivalently the weight polynomial version of DT theory should be the right refinement was suggested independently in $[2,6]$ and $[7]$.

Ongoing work of Nekrasov and Okounkov [26], announced in [27], gives a K-theoretic interpretation of the six-dimensional partition function $Z_{X}$, which, under suitable assumptions, is compatible $^{3}$ with the Hodge-theoretic interpretation given above.

A variant of Theorem 3.5 will be of later use. Recall the interpretation of the spaces $\mathcal{N}_{l, m}$ as spaces of stable representations of the conifold quiver $(\widetilde{Q}, \widetilde{W})$. The specific stability condition depends on a stability parameter $\theta \in \mathbb{R}^{2}$, and the spaces $\mathcal{N}_{l, m}$ arise when $\theta$ takes a value in a certain limiting position [21]. More precisely, there is a set of open cones $\mathcal{C}_{0}, \mathcal{C}_{1}, \ldots \subset \mathbb{R}^{2}$, as in Fig. 1, and moduli spaces $\mathcal{N}_{l, m}^{\theta}$ for $\theta$ inside any of the open chambers $\mathcal{C}_{n}$, such that Theorem 3.2 continues to hold. Also, for each fixed $l, m$, the moduli spaces $\mathcal{N}_{l, m}^{\theta}$ stabilize as $\theta \in \mathcal{C}_{n}$ with

\footnotetext{
${ }^{3}$ Maulik D., Okounkov A., work in progress.
} 


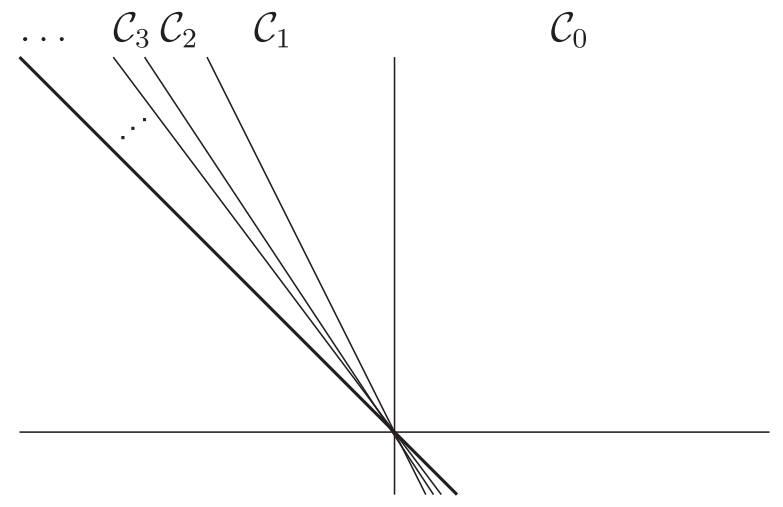

Figure 1. Some of the walls and chambers in the space of stability conditions on the framed conifold quiver, with the thick line representing the D0/D6 wall.

$n \rightarrow \infty$, and agree with the stable pair moduli spaces $\mathcal{N}_{l, m}$. Now for any $\theta$ inside a chamber, we can consider

$$
Z_{X}^{\theta}\left(q, T, t^{\frac{1}{2}}\right)=\sum_{l, m \in \mathbb{Z}} W\left(\mathcal{N}_{l, m}^{\theta}, \Phi_{l, m}^{\theta} ; t^{\frac{1}{2}}\right) T^{l} q^{m}
$$

Theorem 3.9 ([20]). For $n \geq 0$ and $\theta \in \mathcal{C}_{n}$, we have

$$
Z_{X}^{\theta}\left(q, T, t^{\frac{1}{2}}\right)=\prod_{m=1}^{n} \prod_{j=0}^{m-1}\left(1-(-1)^{m} t^{-\frac{m}{2}+\frac{1}{2}+j} q^{m} T\right) .
$$

Beyond the chambers $\mathcal{C}_{n}$ as $n \rightarrow \infty$, is the D0/D6 wall, the other side of which the moduli spaces $\mathcal{N}_{l, m}^{\theta}$ are no longer compact. Immediately on the other side, (infinitesimally) close to the wall, these moduli spaces are in fact the actual Donaldson-Thomas moduli spaces representing rank-one sheaves with trivial determinant (and compactly supported quotient) [21]. In particular, the Hilbert schemes of points of the threefold $X$ make an appearance. The partition functions here include further, refined MacMahon-type factors [20].

Let us return finally to the case $r>1$. First consider the specialization where the torus parameters $q_{1}, q_{2}$ are identified as above. In this case, it is known [12] that the K-theoretic version of the gauge theory partition function reproduces the full Gromow-Witten or reduced Donaldson-Thomas series of the threefold $X$. (I am simplifying here: the threefold $X$ defined in the Introduction is a fibration over $\mathbb{P}^{1}$ by resolved $A_{r-1}$ surface singularitites. For $r>1$, there is in fact a family of such threefolds [11, Fig. 10], whose Gromow-Witten potentials differ by the inclusion of certain framing terms. On the surface side, this difference is accounted for [31] by a change in the integrand in (1), from the trivial class to the class of a power of the determinant line bundle.)

The refined case is largely open for $r>1$. Even for $r=2$, when $X=\mathcal{O}_{\mathbb{P}^{1} \times \mathbb{P}^{1}}(-2,-2)$ is a much-studied local Calabi-Yau threefold, there appear to be significant challenges. The refined topological vertex formalism gives a combinatorial answer [13, Section 5.5], which can be matched with the Nekrasov partition function [13, Section 5.1.1]. The very recent paper [4] uses localization techniques to compute some refined PT invariants, making some assumptions which are justified using [26, 27]. On the quiver side however, the wall crossing picture of [21], used extensively in [20], is a lot more complicated and hardly understood. When trying to match the quiver picture with the geometry, there is no clear understanding how find DT or PT moduli spaces starting from the quiver. It would be very interesting to find a way to calculate all refined PT invariants for this example. 


\section{Purity and Hard Lefschetz}

In this section, I will discuss a result which will be used in the final section. To motivate it, let us assume first that $X=\{d f=0\} \subset N$ is a global degeneracy locus of a smooth function $f: N \rightarrow \mathbb{C}$, and let us moreover assume that $X$ is smooth (and reduced) at every point. Then it follows from the Morse lemma with parameters that the vanishing cycle perverse sheaf is just a local system on $X$ of rank one. Assuming also that $X$ is simply connected, the coefficient system $\Phi$ associated to $f$ is just a shift of the trivial sheaf (Hodge module) $\mathbb{Q}_{X}^{H}$. Assuming finally that $X$ is proper, by Deligne's result, the cohomology $\mathbb{H}^{*}(X, \Phi)$ carries a pure Hodge structure, where the weight filtration agrees with the degree filtration: the weight of a cohomology class equals its degree. In particular, the weight polynomial $W(X, \Phi)$ is just a shift of the topological Poincaré polynomial of $X$. Moreover, if $L \in H^{2}(X, \mathbb{Z})$ is any ample class on $X$, then the maps

$$
\begin{aligned}
L_{k}: \quad \mathbb{H}^{-k}(X, \Phi) & \rightarrow \mathbb{H}^{k}(X, \Phi)(k) \\
\alpha & \mapsto \alpha \cup L^{k}
\end{aligned}
$$

are isomorphisms by the Hard Lefschetz theorem, and can be used to endow $\mathbb{H}^{*}(X, \Phi)$ with an $\mathfrak{s l}(2)$-action.

Our moduli spaces $\mathcal{N}_{l, m}$ are not at all smooth, and the coefficient systems are nontrivial. However, we have the following general result.

Theorem 4.1 ([5]). Let $f: X \rightarrow \mathbb{C}$ be a regular function on a smooth quasi-projective variety. Assume that $X$ carries a $\mathbb{C}^{*}$-action, so that $f$ is equivariant with respect to the weight-1 action of $\mathbb{C}^{*}$ on the base $\mathbb{C}$. Assume also that the critical locus $Z=\{d f=0\}$ is proper, carrying an ample line bundle $\mathcal{L}$.

1. The Hodge structure on the cohomology $\mathbb{H}^{i}\left(Z, \Phi_{f}\right)$ is pure of weight $i$; equivalently, the weight filtration on $\mathbb{H}^{*}\left(X, \Phi_{f}\right)$ agrees with the degree filtration.

2. The ample class $\mathcal{L}$ defines, by cup product as above, Hard Lefschetz-type isomorphisms

$$
\mathcal{L}_{k}: \mathbb{H}^{-k}\left(Z, \Phi_{f}\right) \rightarrow \mathbb{H}^{k}\left(Z, \Phi_{f}\right)(k)
$$

leading to an $\mathfrak{s l}(2)$-action on $\mathbb{H}^{*}\left(\mathcal{N}_{l, m}, \Phi_{l, m}\right)$.

As a consequence, we obtain

Corollary 4.2. For all $l, m$ we have that

1) the Hodge structure on the cohomology $\mathbb{H}^{i}\left(\mathcal{N}_{l, m}, \Phi_{l, m}\right)$ is pure of weight $i$; equivalently, the weight filtration on $\mathbb{H}^{*}\left(\mathcal{N}_{l, m}, \Phi_{l, m}\right)$ agrees with the degree filtration;

2) the ample class $\mathcal{L}_{l, m}$ defines, by cup product as above, Hard Lefschetz-type isomorphisms

$$
\mathcal{L}_{k}: \mathbb{H}^{-k}\left(\mathcal{N}_{l, m}, \Phi_{l, m}\right) \rightarrow \mathbb{H}^{k}\left(\mathcal{N}_{l, m}, \Phi_{l, m}\right)(k)
$$

leading to an $\mathfrak{s l}(2)$-action on $\mathbb{H}^{*}\left(\mathcal{N}_{l, m}, \Phi_{l, m}\right)$.

Proof. By Theorem 3.2, the moduli spaces $\mathcal{N}_{l, m}$ are proper degeneracy loci, and they carry ample line bundles by Remark 3.3. To conclude, it is sufficient to observe that the KlebanovWitten superpotential is linear in each of the variables, so the functions $f_{l, m}$ can be made homogeneous of degree one by acting by $\mathbb{C}^{*}$ on the matrix corresponding to either of the four arrows of the conifold quiver. 
Remark 4.3. For a pure Hodge module $\Phi$ on a proper scheme $X$ of some fixed weight, the cohomology is also pure by the decomposition theorem, and one also has the Lefschetz package [29, Theorem 5.3.1, Remark 5.3.12]. However, the mixed Hodge module $\Phi_{l, m}$ on $\mathcal{N}_{l, m}$ is known not to be of fixed weight in some cases; see Example 4.5 below. There is a similar, but different example of a mixed Hodge module with pure cohomology on a singular projective variety in [8, Section 6].

Example 4.4. Consider first the case $l=1$, corresponding to pairs invariants with the curve class having multiplicity 1. As shown in [28, Section 4.1], the geometry of the corresponding moduli spaces $\mathcal{N}_{1, m}$ is simple: for $m \geq 1, \mathcal{N}_{1, m}$ it is the space of nonzero sections, up to scale, of $\mathcal{O}_{\mathbb{P}^{1}}(m-1)$. Thus

$$
\mathcal{N}_{1, m} \cong \operatorname{Sym}^{m-1}\left(\mathbb{P}^{1}\right) \cong \mathbb{P}^{m-1} .
$$

Moreover, the coefficient system $\Phi_{1, m}$ is (up to shift) just the trivial sheaf, so its (hyper)cohomology is the cohomology of a smooth projective variety with trivial coefficients, carrying in each degree a pure Hodge structure of the correct weight. It is indeed straightforward to check that in $T$-degree $l=1$, the series (6) simply gives the (shifted) weight polynomials (13) of projective spaces in each degree in $q$.

Example 4.5. For the case of the curve class having multiplicity two, in the simplest case $\mathcal{N}_{2,3} \cong \mathbb{P}^{1}$ is still nonsingular. The next space $\mathcal{N}_{2,4}$ is more interesting. The reduced variety underlying $\mathcal{N}_{2,4}$ is isomorphic to $\mathbb{P}^{3}$. As discussed in [28, Section 4.1] however, this cannot be the full answer: the numerical invariant $P_{2,4}$ equals 4 and not -4 , which would be the answer in case the moduli space were just a smooth $\mathbb{P}^{3}$. The moduli space $\mathcal{N}_{2,4}$ is in fact a non-reduced scheme, the tickening of $\mathbb{P}^{3}$ along the embedded quadric $Q \subset \mathbb{P}^{3}$, with Zariski tangent spaces of dimension 4 along the quadric. Via torus localization, this indeed gives the correct value $P_{2,4}=4$. When expanded, the refined expression, the coefficient of $T^{2} q^{4}$ in (6), is $t+2+t^{-1}$, representing the (appropriately shifted) cohomology of the quadric $Q$ itself. I now show, under an assumption, how this answer arises in the present framework.

Recall the function $f_{l, m}$, whose local derivatives cut out $\mathcal{N}_{l, m}$ inside the smooth ambient space $N_{l, m}$. Let us make the assumption that locally around every closed point $p \in Q \subset \mathcal{N}_{2,4} \subset N_{2,4}$ of the quadric, there are local (analytic) coordinates $x_{1}, \ldots, x_{2 n}$ on the smooth $N_{2,4}$, such that the function $f_{2,4}$ is locally of the form $f_{2,4}\left(x_{1}, \ldots, x_{2 n}\right)=x_{3} x_{4}^{2}+\sum_{i=5}^{2 n} x_{i}^{2}$. In this case, the degeneracy locus $\mathcal{N}_{2,4}$ indeed looks around $p$ like a smooth threefold, parametrized by $x_{1}, x_{2}, x_{3}$, with an embedded nilpotent direction with coordinate $x_{4}$ along the codimension one locus $\left\{x_{3}=0\right\}$. While it should be possible to prove this assumption along the lines of [6, Section 3.3], starting from the quiver model, we will not attempt to do that here.

Let us see, under this assumption, what the vanishing cycle module $\Phi_{2,4}$ looks like. This module lives on the reduced degeneracy locus $\mathbb{P}^{3}$. At smooth points, i.e. away from the quadric $Q$, we must have a rank-one local system. At points of $Q$, the local structure is described by Proposition B.1 below. Globally, the summand with nontrivial monodromy can be described as follows. Let $\pi: \widetilde{Q} \rightarrow \mathbb{P}^{3}$ be the double cover of $\mathbb{P}^{3}$ branched along $Q$. Let $U=\mathbb{P}^{3} \backslash Q \stackrel{j}{\hookrightarrow} \mathbb{P}^{3}$ be the inclusion of the complement of $Q$, and $\widetilde{U}=\pi^{-1}(U)$. Then over $U$, we have a mixed Hodge module $L$, with underlying local system of rank one with nontrivial $\mathbb{Z} / 2$ monodromy, such that

$$
\left(\left.\pi\right|_{\widetilde{U}}\right)_{*} \mathbb{Q}_{\widetilde{U}}^{H}=\mathbb{Q}_{\widetilde{U}}^{H} \oplus L
$$

and thus

$$
\pi_{*} \mathbb{Q}_{\widetilde{Q}}^{H}=\mathbb{Q}_{\mathbb{P}^{3}}^{H} \oplus j_{! *} L .
$$


With this notation, we have, as a consequence of Proposition B.1, that

$$
\Phi_{2,4} \cong \mathbb{Q}_{Q}^{H}(1)[2] \oplus j_{! *} L(2)[2] .
$$

In particular, the mixed Hodge module $\Phi_{2,4}$ is a direct sum of pieces with different weights.

On the other hand, $\widetilde{Q}$ is just another quadric, this time in $\mathbb{P}^{4}$. Moreover

$$
\mathbb{H}^{*}\left(\mathbb{P}^{3}, \pi_{*} \mathbb{Q}_{\widetilde{Q}}^{H}\right) \cong H^{*}(\widetilde{Q}, \mathbb{Q}) \cong H^{*}\left(\mathbb{P}^{3}, \mathbb{Q}\right)
$$

Thus, from (8), it follows that

$$
\mathbb{H}^{*}\left(\mathbb{P}^{3}, j_{! *} L\right)=0 .
$$

We finally deduce from (9) that

$$
\mathbb{H}^{*}\left(\mathbb{P}^{3}, \Phi_{2,4}\right) \cong H^{*}\left(Q, \mathbb{Q}_{Q}^{H}(1)[2]\right) .
$$

Thus indeed, the Hodge structure on this cohomology is pure with the correct degrees, and the weight polynomial is $t+2+t^{-1}$ as demanded by our formula (6).

Exercise 4.6. Study the explicit geometry of the next moduli space $\mathcal{N}_{2,5}$ and the mixed Hodge module $\Phi_{2,5}$ in similar detail.

\section{$5 \quad$ Vector spaces underlying partition functions}

The aim in this section is to investigate whether the relationship (7) can be used to study the vector spaces underlying these partition functions. As discussed before, on the left hand side of (7), the four-dimensional partition function $Z_{\mathbb{C}^{2}, r=1}\left(q_{1}, q_{2}, \Lambda\right)$ is the graded character of an actual triply-graded vector space

$$
\bigoplus_{k \geq 0} H^{0}\left(\mathcal{O}_{S^{k}\left(\mathbb{C}^{2}\right)}\right) \cong S^{*} \mathbb{C}[x, y]
$$

On the right hand side of (7), we have weight polynomials. In general, forming the weight polynomial involves taking an Euler characteristic: in the defining formula (5), there can be cancellation between different pieces of cohomology with the same weight (see Examples A.2 and A.3). However, it is clear from the formula that this cannot occur for pure Hodge structures, where the weight filtration is trivial, agreeing with the obvious grading of cohomology by degree. Indeed, by Corollary 4.2(1), all these cohomologies carry pure Hodge structures, and thus

$$
Z_{X}(q, T, t)=\operatorname{char}_{\left(\mathbb{C}^{*}\right)^{3}} \bigoplus_{l, m} \mathbb{H}^{*}\left(\mathcal{N}_{l, m}, \Phi_{l, m}\right)
$$

is also the graded character of a triply-graded (super) vector space, graded by the curve degree $l$, the point degree $m$ and the cohomology degree.

Given that the four-dimensional partition function is the graded character of a symmetric space of a vector space, the inverse operation in (7) has a natural interpretation: we obtain

$$
Z_{X}(q, T, t)=\operatorname{char}_{\left(\mathbb{C}^{*}\right)^{3}} \Lambda^{*} \mathbb{C}[x, y]
$$

under a grading where $x, y$ are viewed as odd variables (introducing signs into the Hilbert series) of weights $\left(1,0, \pm \frac{1}{2}\right)$, and the outer exterior operation has weight $(1,1,0)$. The following is the main result of our paper. 
Theorem 5.1. There exists a natural $\mathrm{GL}(2) \times \mathbb{C}^{*}$-equivariant isomorphism

$$
\bigoplus_{l, m} \mathbb{H}^{*}\left(\mathcal{N}_{l, m}, \Phi_{l, m}\right) \cong \Lambda^{*} \mathbb{C}[x, y]
$$

of (super) vector spaces.

Proof. Our computation of the torus characters means that we certainly have a triply graded, in other words $\left(\mathbb{C}^{*}\right)^{3}$-equivarient isomorphism.

Return to the four-dimensional partition function for a moment. The $T=\left(\mathbb{C}^{*}\right)^{2}$-action on $\mathbb{C}^{2}$ is part of a $\mathrm{GL}(2)$-action; the element $\left(\begin{array}{ll}0 & 1 \\ 1 & 0\end{array}\right)$ acts on the partition function simply by interchanging $x$ and $y$. Looking at the change of variables in Corollary 3.7, we see that this corresponds to mapping $t^{\frac{1}{2}} \mapsto t^{-\frac{1}{2}}$, while keeping the other variables fixed, in other words to Poincaré duality on the individual cohomologies $\mathbb{H}^{*}\left(\mathcal{N}_{l, m}, \Phi_{l, m}\right)$, for every fixed $l, m$. This then means that we can enhance the corresponding $\left(\mathbb{C}^{*}\right)^{2}$-action on the left hand side of $(11)$ to a $\mathrm{GL}(2)$-action, integrating the $\mathfrak{s l}(2)$-action coming from Corollary $4.2(2)$, with Weyl element given by the action of the Hard Lefschetz isomorphism. We then have compatible GL $(2) \times \mathbb{C}^{*}$ actions on all the spaces in (10), (11), compatible with the isomorphisms.

Before I discuss (11) any further, let me make a digression. Recall Theorem 3.9, computing a six-dimensional partition function depending on a parameter $\theta$. Checking the effect of the truncation, the following extension of Theorem 5.1 turns out to be compatible with the partition function.

Theorem 5.2. For $n \geq 0$ and $\theta \in \mathcal{C}_{n}$, there exists a $\mathrm{GL}(2) \times \mathbb{C}^{*}$-equivarient isomorphism

$$
\bigoplus_{l, m} \mathbb{H}^{*}\left(\mathcal{N}_{l, m}^{\theta}, \Phi_{l, m}^{\theta}\right) \cong \Lambda^{*} \mathbb{C}[x, y]_{n-1}
$$

of (super) vector spaces, where $\mathbb{C}[x, y]_{n}$ denotes the space of polynomials of total degree at most $n$.

I want to address the issue of naturality of the isomorphisms (11), (12). Recall one final time the conifold quiver with potential $(Q, W)$ and its close relative, the framed quiver $(\widetilde{Q}, \widetilde{W})$. In a recent paper [16], Kontsevich and Soibelman introduce an associative algebra $\mathcal{H}(Q, W)$, the (critical) cohomological Hall algebra of the quiver $(Q, W)$, built from all representations of the quiver $Q$, the vanishing cycle complex of the function $\operatorname{Tr}(W)$ defined on these spaces of representations, and equivariant cohomology with respect to the natural action of products of general linear groups.

The associative product on $\mathcal{H}(Q, W)$ is defined in [16] by using a version of the standard diagram which essentially fuses two representations $\mathcal{R}_{i}$ of $(Q, W)$ into a third one: an exact sequence

$$
0 \rightarrow \mathcal{R}_{2} \rightarrow \mathcal{R}_{3} \rightarrow \mathcal{R}_{1} \rightarrow 0
$$

of representations of $(Q, W)$ gives the multiplication

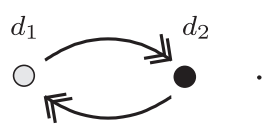

$\mathcal{R}_{1}$

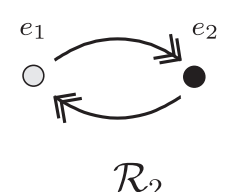

$\mathcal{R}_{2}$

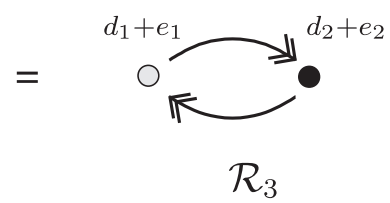

$\mathcal{R}_{3}$

The same construction should turn the space on the right hand side of (11), the sum of cohomologies of representation spaces of the quiver $(\widetilde{Q}, \widetilde{W})$, into modules over the algebra $\mathcal{H}(Q, W)$. 
The point is that the dimension on the extra vertex in $\widetilde{Q}$ is always one, and this is unchanged by the operations which attach representations $\mathcal{R}$ of $(Q, W)$ : an exact sequence

$$
0 \rightarrow \mathcal{R} \rightarrow \widetilde{\mathcal{R}}_{2} \rightarrow \widetilde{\mathcal{R}}_{1} \rightarrow 0
$$

of representations of $(\widetilde{Q}, \widetilde{W})$ should give the multiplication

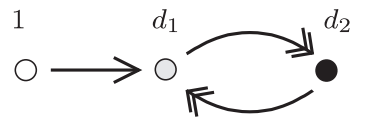

$\widetilde{\mathcal{R}}_{1}$

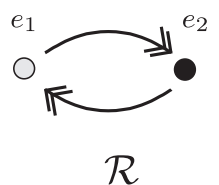

$\mathcal{R}$
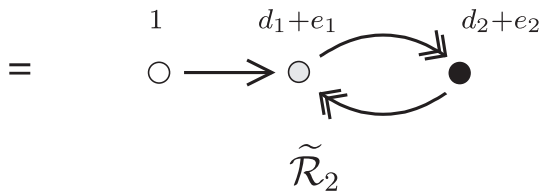

See the recent preprint [10, Section 2] for a similar, possibly not unrelated, perhaps mirror, idea of how to construct representations of the conifold CoHA (algebra of BPS states).

The structure of the algebra $\mathcal{H}(Q, W)$ is not known explicitly at present. But the most natural extension of the ideas above is that there should exist unique isomorphisms (11), (12) of $\mathcal{H}(Q, W)$-modules, exhibiting $\Lambda^{*} \mathbb{C}[x, y]$ as a Verma-type module of the algebra $\mathcal{H}(Q, W)$ and $\Lambda^{*} \mathbb{C}[x, y]_{n}$ as finite-dimensional highest-weight quotients.

Remark 5.3. As a final point, notice that the fact that we could interpret both the fourand the six-dimensional partition function as graded characters of vector spaces (as opposed to virtual torus representations) depended on both sides on a fortuitous lack of cancellation. On the six-dimensional side, this is provided by the purity of cohomologies of mixed Hodge modules of vanishing cycles. On the four-dimensional side, (2) involves an index, in other words an alternating sum of cohomologies; however, as discussed around (3), there is no higher cohomology, leading to an actual, rather than virtual, torus representation. I can see no direct connection between these facts, but it would be really interesting if one existed.

\section{A Weights on cohomology}

Since the weight filtration plays an important role in the paper, here we collect some conventions for weights. Recall that by Deligne's theorem, the rational cohomology $H^{*}(X, \mathbb{Q})$ of a variety $X$, not necessarily smooth or projective, admits a mixed Hodge structure: it carries a weight filtration, so that the associated graded pieces carry pure Hodge structures. If $X$ is smooth and projective, then the weight filtration is trivial: it coincides with the degree filtration. More generally, the smoothness or projectivity on $X$ imply estimates on the weights appearing in the cohomology.

The modern way to view Deligne's mixed Hodge structure is to first consider, for a general scheme $X$, the category of pure Hodge modules $\operatorname{HM}(X)$, sitting inside a category of mixed Hodge modules $\operatorname{MHM}(X)$; objects in these categories are complicated, represented by a perverse topological $\mathbb{Q}$-sheaf, an associated complex algebraic gadget (a $D$-module) and some compatibility data. Pure Hodge modules are direct sums of Hodge modules, each of which has a fixed weight; mixed Hodge modules are extensions of pure Hodge modules. The next step is to form complexes of mixed Hodge modules, to arrive at the bounded derived category $\mathcal{D}^{b} \operatorname{MHM}(X)$. For an object $\mathcal{F} \in \mathcal{D}^{b} \operatorname{MHM}(X), \mathcal{F}[k]$ denotes the complex $\mathcal{F}$ shifted by $k$ places.

Maps $f: X \rightarrow Y$ between algebraic varieties define pushforward maps between the corresponding derived categories; there are two types of pushforward $f_{*}$ and $f_{!}$, corresponding in the case the target $Y=\{P\}$ is a point to taking cohomology on $X$ and cohomology with compact support. The categories of Hodge modules and mixed Hodge modules over a point are equivalent to the categories of pure and mixed Hodge structures; thus cohomologies of mixed Hodge modules on arbitrary $X$ carry mixed Hodge structures. There are also two types of pullback maps $f^{*}$ and $f^{!}$. 
Every smooth variety $X$ carries a canonical pure Hodge module $\mathbb{Q}_{X}^{H} \in \operatorname{HM}(X)$ of weight $2 \operatorname{dim} X$. Proper pushforward preserves purity, so we obtain that if $X$ is moreover proper, then $H^{*}\left(X, \mathbb{Q}_{X}^{H}\right)$ is pure; this is simply the classical cohomology of $X$. For the projective line, we have

$$
H^{*}\left(\mathbb{P}^{1}, \mathbb{Q}_{\mathbb{P}^{1}}^{H}\right) \cong \mathbb{Q} \oplus \mathbb{Q}(-1)[-2]
$$

here $\mathbb{Q}(-1)$ is the Tate Hodge structure, a one-dimensional pure Hodge structure of weight 2 (the analogue of the motive $\mathbb{L}$ in the motivic ring of varieties). The shift [-2] corresponds to the fact that it arises as the second cohomology. For an arbitrary complex of mixed Hodge modules $\mathcal{F} \in \mathcal{D}^{b} \operatorname{MHM}(X)$, we let $\mathcal{F}(i)$ denote the tensor product of $\mathcal{F}$ with $f^{*} \mathbb{Q}(i)$, where $f: X \rightarrow\{P\}$ is the structure morphism. If $\mathcal{F}$ is pure of weight $k$, then $\mathcal{F}(i)$ is pure of weight $k-2 i$.

To treat odd-dimensional varieties, it is convenient to adjoin to the category of mixed Hodge structures a half-Tate object $\mathbb{Q}\left(\frac{1}{2}\right)$, of weight -1 , with the property that $\mathbb{Q}\left(\frac{1}{2}\right) \otimes \mathbb{Q}\left(\frac{1}{2}\right) \cong \mathbb{Q}(1)$; see [16, Section 3.4]. Then for any smooth projective $X$, the cohomology

$$
H^{*}\left(X, \mathbb{Q}_{X}^{H}(\operatorname{dim} X / 2)[\operatorname{dim} X]\right)
$$

lives in palindromic degrees $-\operatorname{dim} X, \ldots, \operatorname{dim} X$ with the same weights. The pullback to any variety $X$ of $\mathbb{Q}\left(\frac{1}{2}\right)$ under the structure morphism will still be denoted by $\mathbb{Q}\left(\frac{1}{2}\right)$; we denote by $\widetilde{\operatorname{MHM}}(X)$ the extended category of mixed Hodge modules on a variety $X$.

Given a complex of mixed Hodge modules $\mathcal{F} \in \mathcal{D}^{b} \widehat{\mathrm{MHM}}(X)$, consider the compactly supported (hyper)cohomology $\mathbb{H}_{c}^{*}(X, \mathcal{F})$ with its weight filtration $W_{n}$. We can consider the weight polynomial (sometimes called Serre polynomial)

$$
W\left(X, \mathcal{F} ; t^{\frac{1}{2}}\right)=\sum_{n \in \mathbb{Z}} t^{\frac{n}{2}} \sum_{i \in \mathbb{Z}}(-1)^{i} \operatorname{dim} \operatorname{Gr}_{n}^{W} \mathbb{H}_{c}^{i}(X, \mathcal{F}) \in \mathbb{Z}\left[t^{ \pm \frac{1}{2}}\right] .
$$

Then

$$
W\left(X, \mathcal{F}(i)[j] ; t^{\frac{1}{2}}\right)=(-1)^{j} t^{-\frac{i}{2}} W\left(X, \mathcal{F} ; t^{\frac{1}{2}}\right) .
$$

A very important property of the weight polynomial is its additivity under stratifications: because of the long exact sequence in cohomology and its compatibility with Hodge and weight filtrations, the weight polynomial behaves well under decompositions $X=(X \backslash Z) \cup Z$, where $Z \subset X$ is a closed subvariety.

Example A.1. To start with,

$$
W\left(\mathbb{A}^{n}, \mathbb{Q}_{\mathbb{A}^{1}}^{H} ; t^{\frac{1}{2}}\right)=t^{n}
$$

as the only nonzero compactly supported cohomology of $\mathbb{A}^{n}$ is one-dimensional of weight $2 n$ in degree $2 n$. Also

$$
W\left(\mathbb{P}^{1}, \mathbb{Q}_{\mathbb{P}^{1}}^{H} ; t^{\frac{1}{2}}\right)=1+t
$$

and so

$$
W\left(\mathbb{P}^{1}, \mathbb{Q}_{\mathbb{P}^{1}}^{H}(1 / 2)[1] ; t^{\frac{1}{2}}\right)=-\left(t^{\frac{1}{2}}+t^{\frac{1}{2}}\right) ;
$$

more generally,

$$
W\left(\mathbb{P}^{n}, \mathbb{Q}_{\mathbb{P} n}^{H}(n / 2)[n] ; t^{\frac{1}{2}}\right)=(-1)^{n}\left(t^{-\frac{n}{2}}+\cdots+t^{\frac{n}{2}}\right)=(-1)^{n} \frac{t^{\frac{n+1}{2}}-t^{-\frac{n+1}{2}}}{t^{\frac{1}{2}}-t^{-\frac{1}{2}}} .
$$


Even more generally, for a smooth projective variety $X, H^{i}(X, \mathbb{Q})$ is of weight $i$, so we have

$$
W\left(X, \mathbb{Q}_{X}^{H} ; t^{\frac{1}{2}}\right)=\sum_{n=0}^{2 \operatorname{dim} X}(-1)^{i} b_{i}(X) t^{\frac{i}{2}},
$$

where the $b_{i}$ are the Betti numbers of $X$.

Example A.2. For $E$ an elliptic curve,

$$
W\left(E, \mathbb{Q}_{E}^{H} ; t^{\frac{1}{2}}\right)=1-2 t^{\frac{1}{2}}+t .
$$

Consider now the complex of Hodge structures (complex of Hodge modules on a point $P$ ) $\mathcal{F}=H^{*}(E, \mathbb{Q}) \oplus \mathbb{Q}(1 / 2)^{\oplus 2}$. This has the previous pieces $H^{2 i}(E, \mathbb{Q})[-2 i]$ in weight $2 i$, and $H^{1}(E)[-1] \oplus \mathbb{Q}^{\oplus 2}$ in weight 1 . Thus, its weight polynomial is

$$
W\left(P, \mathcal{F} ; t^{\frac{1}{2}}\right)=\left(1-2 t^{\frac{1}{2}}+t\right)+2 t^{\frac{1}{2}}=1+t,
$$

indistinguishable from the weight polynomial coming from the cohomology of $\mathbb{P}^{1}$ with constant coefficients. This is the kind of cancellation that cannot occur under the purity statement of Theorem 4.1.

Exercise A.3. For a more geometric example of cancellation, let $X$ be the blowup of a point in $\mathbb{A}^{1} \times \mathbb{C}^{*}$. Show that its weight polynomial with constant coefficients is

$$
W\left(X, \mathbb{Q}_{X}^{H} ; t^{\frac{1}{2}}\right)=t^{2},
$$

indistinguishable from that of $\mathbb{A}^{2}$, even though $X$ obviously has odd cohomology.

\section{B A vanishing cycle computation}

We compute the vanishing cycle mixed Hodge module of the function $f(x, y)=x y^{2}$ on $X=\mathbb{C}^{2}$. This sort of computation is presumably trivial for the experts, but I couldn't find the answer in the literature in a form needed above.

Start with the inclusions

$$
P=\{0\} \in Z=\{d f=0\}_{\text {red }} \subset Y=\{f=0\}_{\text {red }} \subset X,
$$

with $P$ being the origin, $Z$ the $x$-axis and $Y$ the union of the axes. On the smooth $X=\mathbb{C}^{2}$, we have $\mathbb{Q}_{X}^{H}[2] \in \operatorname{MHM}(X)$, a pure Hodge module of weight 2 . On the hypersurface $Y$, we have the mixed Hodge module of vanishing cycles $\varphi_{f} \mathbb{Q}_{X}^{H} \in \operatorname{MHM}(Y)$. There is an exact sequence of MHM's on $Y$

$$
0 \rightarrow \mathbb{Q}_{Y}^{H}[1] \rightarrow \psi_{f} \mathbb{Q}_{X}^{H}[2] \rightarrow \varphi_{f} \mathbb{Q}_{X}^{H}[2] \rightarrow 0 .
$$

The weight filtration of $\mathbb{Q}_{Y}^{H}[2]$ has $I C_{Y}^{H}$ in weight 1 , then $i_{*} \mathbb{Q}_{P}^{H}$ in weight 0 , with $i: P \rightarrow Y$ denoting the inclusion of the origin $P$. Now this injects into $\psi_{f} \mathbb{Q}_{X}^{H}[2]$, and the action of the monodromy operator $N$ on $\psi_{f} \mathbb{Q}_{X}^{H}[2]$ has to satisfy Hard Lefschetz. The nearby cycle over the punctured $x$-axis consists of two points. Correspondingly, there is a local system $M$ on the smooth locus $j: U \rightarrow Y$, which is trivial over the punctured $y$-axis, and is of rank two over the punctured $x$-axis. Then $\psi_{f} \mathbb{Q}_{X}^{H}[3]$ has to look as follows: in weight $2: i_{*} \mathbb{Q}_{P}^{H}(-1)$; in weight 1 : $I C_{Y}^{H}(M)$; in weight $0: i_{*} \mathbb{Q}_{P}^{H}$. We have $N^{2}=0$, but $N$ is nonzero, taking the weight-2 piece isomorphically to the weight-0 piece. The cokernel of the inclusion $\mathbb{Q}_{Y}^{H}[2] \rightarrow \psi_{f} \mathbb{Q}_{X}^{H}[3]$ is $\varphi_{f} \mathbb{Q}_{X}^{H}[3]$. We deduce that $\varphi_{f} \mathbb{Q}_{X}^{H}[3]$ has weight filtration as follows. In weight 2 , the associated graded is $i_{*} \mathbb{Q}_{P}^{H}(-1)$; in weight 1 , it is $I C_{Z}^{H}(L)$, where $L$ is the nontrivial rank-one local system on the punctured $x$-axis with $\mathbb{Z} / 2$ monodromy. Note finally that the weight filtration splits, either because of duality, or due to the fact that the monodromy-invariant part $\varphi_{f, 1} \mathbb{Q}_{X}^{H}[2] \cong i_{*} \mathbb{Q}^{H}(-1)$ is always a direct summand. In summary, 
Proposition B.1. In the category $\operatorname{MHM}(Z)$, the module $\varphi_{f} \mathbb{Q}_{X}^{H}[2]$ splits as a direct sum

$$
\varphi_{f} \mathbb{Q}_{X}^{H}[2] \cong i_{*} \mathbb{Q}_{P}^{H}(-1) \oplus I C_{Z}^{H}(L),
$$

with weights 2 and 1 respectively.

\section{Acknowledgements}

I wish to thank Jim Bryan, Lotte Hollands, Dominic Joyce, Davesh Maulik, Geordie Williamson and especially Ian Grojnowski for comments and discussions. This research was supported by EPSRC Programme Grant EP/I033343/1, and by a Fellowship from the Alexander von Humboldt Foundation. Part of this paper was prepared while I was visiting the Department of Mathematics, Freie Universität Berlin; I wish to thank them and especially Klaus Altmann for hospitality.

\section{References}

[1] Behrend K., Donaldson-Thomas type invariants via microlocal geometry, Ann. of Math. (2) 170 (2009), 1307-1338, math.AG/0507523.

[2] Behrend K., Bryan J., Szendrői B., Motivic degree zero Donaldson-Thomas invariants, Invent. Math., to appear, arXiv:0909.5088.

[3] Bridgeland T., Hall algebras and curve-counting invariants, J. Amer. Math. Soc. 24 (2011), 969-998, arXiv:1002.4374.

[4] Choi J., Katz S., Klemm A., The refined BPS index from stable pair invariants, arXiv:1210.4403.

[5] Davison B., Maulik D., Schuermann J., Szendrői B., Purity for graded potentials and cluster positivity, unpublished.

[6] Dimca A., Szendrői B., The Milnor fibre of the Pfaffian and the Hilbert scheme of four points on $\mathbb{C}^{3}$, Math. Res. Lett. 16 (2009), 1037-1055, arXiv:0904.2419.

[7] Dimofte T., Gukov S., Refined, motivic, and quantum, Lett. Math. Phys. 91 (2010), 1-27, arXiv:0904.1420.

[8] Efimov A.I., Quantum cluster variables via vanishing cycles, arXiv:1112.3601.

[9] Gopakumar R., Vafa C., M-theory and topological strings - I, hep-th/9809187.

[10] Gukov S., Stosic M., Homological algebra of knots and BPS states, arXiv:1112.0030.

[11] Hollowood T., Iqbal A., Vafa C., Matrix models, geometric engineering and elliptic genera, J. High Energy Phys. 2008 (2008), no. 3, 069, 81 pages, hep-th/0310272.

[12] Iqbal A., Kashani-Poor A.K., SU(N) geometries and topological string amplitudes, Adv. Theor. Math. Phys. 10 (2006), 1-32, hep-th/0306032.

[13] Iqbal A., Kozçaz C., Vafa C., The refined topological vertex, J. High Energy Phys. 2009 (2009), no. 10, 069, 58 pages, hep-th/0701156.

[14] Katz S., Klemm A., Vafa C., Geometric engineering of quantum field theories, Nuclear Phys. B 497 (1997), 173-195, hep-th/9609239.

[15] Klebanov I.R., Witten E., Superconformal field theory on threebranes at a Calabi-Yau singularity, Nuclear Phys. B 536 (1999), 199-218, hep-th/9807080.

[16] Kontsevich M., Soibelman Y., Cohomological Hall algebra, exponential Hodge structures and motivic Donaldson-Thomas invariants, Commun. Number Theory Phys. 5 (2011), 231-352, arXiv:1006.2706.

[17] Kontsevich M., Soibelman Y., Stability structures, motivic Donaldson-Thomas invariants and cluster transformations, arXiv:0811.2435.

[18] Losev A., Moore G., Nekrasov N., Shatashvili S., Four-dimensional avatars of two-dimensional RCFT, Nuclear Phys. B Proc. Suppl. 46 (1996), 130-145, hep-th/9509151.

[19] Maulik D., Nekrasov N., Okounkov A., Pandharipande R., Gromov-Witten theory and Donaldson-Thomas theory. I, Compos. Math. 142 (2006), 1263-1285, math.AG/0312059. 
[20] Morrison A., Mozgovoy S., Nagao K., Szendrői B., Motivic Donaldson-Thomas invariants of the conifold and the refined topological vertex, Adv. Math. 230 (2012), 2065-2093, arXiv:1107.5017.

[21] Nagao K., Nakajima H., Counting invariant of perverse coherent sheaves and its wall-crossing, Int. Math. Res. Not. 2011 (2011), 3885-3938, arXiv:0809.2992.

[22] Nakajima H., Yoshioka K., Instanton counting on blowup. I. 4-dimensional pure gauge theory, Invent. Math. 162 (2005), 313-355, math.AG/0306198.

[23] Nakajima H., Yoshioka K., Instanton counting on blowup. II. K-theoretic partition function, Transform. Groups 10 (2005), 489-519, math.AG/0505553.

[24] Nekrasov N.A., Seiberg-Witten prepotential from instanton counting, Adv. Theor. Math. Phys. 7 (2003), 831-864, hep-th/0206161.

[25] Nekrasov N.A., Okounkov A., Seiberg-Witten theory and random partitions, in The Unity of Mathematics, Progr. Math., Vol. 244, Birkhäuser Boston, Boston, MA, 2006, 525-596, hep-th/0306238.

[26] Nekrasov N.A., Okounkov A., The index of M-theory, work in progress.

[27] Okounkov A., The index and the vertex, Talk at Brandeis-Harvard-MIT-Northeastern Joint Mathematics Colloquium, December 1, 2011.

[28] Pandharipande R., Thomas R.P., Curve counting via stable pairs in the derived category, Invent. Math. 178 (2009), 407-447.

[29] Saito M., Modules de Hodge polarisables, Publ. Res. Inst. Math. Sci. 24 (1988), 849-995.

[30] Szendrői B., Non-commutative Donaldson-Thomas invariants and the conifold, Geom. Topol. 12 (2008), 1171-1202, arXiv:0705.3419.

[31] Tachikawa Y., Five-dimensional Chern-Simons terms and Nekrasov's instanton counting, J. High Energy Phys. 2004 (2004), 050, 13 pages, hep-th/0401184. 